\title{
OPTIMAL LQ-FEEDBACK CONTROL FOR A CLASS OF FIRST-ORDER HYPERBOLIC DISTRIBUTED PARAMETER SYSTEMS
}

\author{
Ilyasse Aksikas $^{1}$, Joseph J. Winkin ${ }^{2}$ And Denis Dochain ${ }^{3}$
}

\begin{abstract}
The Linear-Quadratic (LQ) optimal control problem is studied for a class of first-order hyperbolic partial differential equation models by using a nonlinear infinite-dimensional (distributed parameter) Hilbert state-space description. First the dynamical properties of the linearized model around some equilibrium profile are studied. Next the LQ-feedback operator is computed by using the corresponding operator Riccati algebraic equation whose solution is obtained via a related matrix Riccati differential equation in the space variable. Then the latter is applied to the nonlinear model, and the resulting closed-loop system dynamical performances are analyzed.
\end{abstract}

Mathematics Subject Classification. 49J20, 93B52, 34K30, 47H06, 34K20

Received March 27, 2007. Revised July 30, 2007.

Published online February 7, 2008.

\section{INTRODUCTION}

Phenomena in many chemical processes include transport processes that are described by partial differential equations (PDE's): see e.g. $[12,15,18]$. When diffusive transport is negligible and convective transport is dominant, processes are described by first-order hyperbolic PDE's. The class of such processes includes plug flow reactors.

The dynamical analysis and (optimal) control of distributed parameter systems modeled by hyperbolic PDE's are important questions that have received a lot of attention in the system and control literature: see e.g. $[7,8,20]$ for the study of such questions from the applied mathematics point of view, and e.g. $[12,16,24]$ for applications to process control. In most practical situations the original infinite-dimensional systems described by PDE's are spatially discretized to obtain finite-dimensional approximate models, and then finite-dimensional controllers are designed and implemented. However, the latter approach neglects the distributed nature of the original system; this may lead to inappropriate control design that neglects the dynamical properties of the infinite-dimensional system and may impose some limitations on the controller performances.

In $[4,5]$, the Linear-Quadratic (LQ) problem is studied for a nonisothermal plug flow reactor model that represents a particular process described by first-order hyperbolic PDE's. In this paper, the LQ-optimal control

\footnotetext{
Keywords and phrases. First-order hyperbolic PDE's, infinite-dimensional systems, LQ-optimal control, stability, optimality.

1 Department of Chemical and Materials Engineering, University of Alberta, Edmonton, Alberta, T6G 2G6, Canada; aksikas@ualberta.ca

2 Department of Mathematics, University of Namur (FUNDP), 8 Rempart de la Vierge, 5000 Namur, Belgium;

Joseph. Winkin@fundp.ac. be

3 CESAME, Université Catholique de Louvain, 4-6 avenue G. Lemaître, 1348 Louvain-la-Neuve, Belgium;

dochain@csam.ucl.ac.be
} 
problem is studied for a more general class of first-order hyperbolic PDE models by using a nonlinear infinitedimensional Hilbert state-space description. Section 2 describes both the nonlinear model and linearized model of this class of systems. Section 3 focuses on dynamical properties (exponential stability, reachability and observability) of the linearized model. The optimal control design is the object of Section 4. An LQ-optimal feedback is computed by using the corresponding operator Riccati algebraic equation, whose solution is obtained via a related matrix Riccati differential equation. Then, in Section 5, this LQ-optimal feedback is applied to the nonlinear model, and the resulting closed-loop system dynamical performances are analyzed. Criteria are given that guarantee that the closed-loop system is asymptotically stable. Moreover optimality is proved with respect to a modified cost criterion.

\section{MODELS DESCRIPTION}

\subsection{Nonlinear model}

Let us consider the following semilinear first-order hyperbolic PDE system in one spatial dimension:

$$
\begin{gathered}
\frac{\partial x}{\partial t}=-\frac{\partial x}{\partial z}+f(x, u) \\
y=h(x)
\end{gathered}
$$

subject to the boundary condition: for all $t \geq 0$ :

$$
x(0, t)=b
$$

and the initial condition: for almost all $z \in[0,1]$ :

$$
x(z, 0)=x_{0}(z)
$$

where $z \in[0,1]$ (without loss of generality) and $t \in[0, \infty)$ denote the spatial variable and the time variable respectively, $x(t):=\left[x_{1}(\cdot, t), \ldots x_{n}(\cdot, t)\right]^{T} \in H:=L^{2}(0,1)^{n}, u(t):=\left[u_{1}(\cdot, t), \ldots u_{m}(\cdot, t)\right]^{T} \in U:=L^{2}(0,1)^{m}$, and $y(t):=\left[y_{1}(\cdot, t), \ldots y_{p}(\cdot, t)\right]^{T} \in Y:=L^{2}(0,1)^{p}$ denote the vectors of state (input, output, respectively) variables at time $t$, the functions $f:=\left[f_{1} \ldots f_{n}\right]: H \times U \longrightarrow H$ and $h:=\left[h_{1} \ldots h_{p}\right]: H \longrightarrow Y$ are continuous vector functions, $b \in \mathbb{R}^{n}$ is a constant (column) vector and $x_{0}$ is in $H$.

Let us assume that the system (2.1)-(2.2) has at least one equilibrium profile $\left(x_{e}, u_{e}\right) \in H \times U$, i.e. $\left(x_{e}, u_{e}\right)$ is a solution of the following ordinary differential equation

$$
\frac{\mathrm{d} x_{e}}{\mathrm{~d} z}=f\left(x_{e}(z), u_{e}(z)\right), \quad x_{e}(0)=b
$$

Remark 2.1. (a) In this paper, it will be assumed that the hyperbolic PDE (2.1), together with the boundary condition (2.3) and the initial condition (2.4) is well posed, i.e. that, with any fixed input function $u$, the state trajectories exist such that, for all $t \geq 0, x(t)$ is in $H$. See e.g. [8], Chapter 3, for more detail concerning the existence of solutions to semilinear and quasilinear systems; see also [22,23] for the analysis of the state trajectories of such models, by means of general mathematical descriptions involving abstract differential equations on Banach or Hilbert spaces. In addition, specific existence conditions for the state trajectories will be established in Section 5, for such hyperbolic PDE system under LQ-optimal state feedback.

(b) If the function $f$ is assumed to be Lipschitz continuous on its domain, then the system (2.1)-(2.2) has a unique equilibrium profile $x_{e}$, corresponding to a fixed input function $u_{e}$. This applies in particular to the nonisothermal plug flow reactor studied in [3-5].

In what follows, although we do not necessarily linearize the system (2.1)-(2.2) around the trivial equilibrium $(0,0)$, we shall keep for simplicity $x$ and $u$ as notations for the new variables $x-x_{e}$ and $u-u_{e}$, respectively. 


\subsection{Linearized model}

The linearization of the system (2.1)-(2.2) around an equilibrium profile leads to the following linear timeinvariant infinite-dimensional state-space system on the Hilbert space $H$ :

$$
\left\{\begin{array}{l}
\dot{x}(t)=A x(t)+B u(t), x(0)=x_{0} \in H \\
y(t)=C x(t) .
\end{array}\right.
$$

Here $A$ is the linear (unbounded) operator defined on its domain:

$$
D(A)=\left\{x \in H: x \text { is a.c., } \frac{\mathrm{d} x}{\mathrm{~d} z} \in H \text { and } x(0)=0\right\}
$$

(where a.c. means that the function $x$ is absolutely continuous) by

$$
A=\left[\begin{array}{cccc}
-\frac{\mathrm{d} .}{\mathrm{d} z}-\alpha_{1,1} I & -\alpha_{1,2} I & \ldots & -\alpha_{1, n} I \\
-\alpha_{2,1} I & -\frac{\mathrm{d}}{\mathrm{d} z}-\alpha_{2,2} I & \ldots & -\alpha_{2, n} I \\
\vdots & \vdots & \ddots & \vdots \\
-\alpha_{n, 1} I & -\alpha_{n, 2} I & \ldots & -\frac{\mathrm{d} .}{\mathrm{d} z}-\alpha_{n, n} I
\end{array}\right]
$$

where $I$ denotes the identity operator on $L^{2}(0,1)$. The operators $B \in \mathcal{L}(U, H)$ and $C \in \mathcal{L}(H, Y)$ are the bounded linear operators defined by

$$
B u=\left[\begin{array}{cccc}
\beta_{1,1} I & \beta_{1,2} I & \ldots & \beta_{1 m} I \\
\beta_{2,1} I & \beta_{2,2} I & \ldots & \beta_{2, m} I \\
\vdots & \vdots & \ddots & \vdots \\
\beta_{n, 1} I & \beta_{n, 2} I & \ldots & \beta_{n, m} I
\end{array}\right]\left[\begin{array}{c}
u_{1} \\
u_{2} \\
\vdots \\
u_{m}
\end{array}\right]
$$

and

$$
C x=\left[\begin{array}{cccc}
w_{1,1} I & w_{1,2} I & \ldots & w_{1, n} I \\
w_{2,1} I & w_{2,2} I & \ldots & w_{2, n} I \\
\vdots & \vdots & \ddots & \vdots \\
w_{p, 1} I & w_{n, 2} I & \ldots & w_{p, n} I
\end{array}\right]\left[\begin{array}{c}
x_{1} \\
x_{2} \\
\vdots \\
x_{n}
\end{array}\right]
$$

Denoting by $\underline{k}$ the set of positive integers $\{1, \ldots, k\}$, the functions $\alpha_{i, j}(z), i, j \in \underline{n}, \beta_{i, j}(z), i \in \underline{n}, j \in \underline{m}$ and $w_{i, j}(z), i \in \underline{p}, j \in \underline{n}$ correspond to the Jacobians of the nonlinear functions $f$ and $h$ of the model equations evaluated at the chosen equilibrium profile and are given by

$$
\alpha_{i, j}=-\frac{\partial f_{i}}{\partial x_{j}}\left(x_{e}, u_{e}\right), \beta_{i, j}=\frac{\partial f_{i}}{\partial u_{j}}\left(x_{e}, u_{e}\right), w_{i, j}=\frac{\partial h_{i}}{\partial x_{j}}\left(x_{e}\right) .
$$

Remark 2.2. It is assumed that all the functions $\alpha_{i, j}, \beta_{i, j}$ and $w_{i, j}$ are in $L^{\infty}(0,1)$. These conditions guarantee in particular that the linear operators $B$ and $C$ defined above are bounded on $U$ and $H$ respectively.

Let us denote by $\mathcal{M}, \mathcal{N}$ and $\mathcal{C}$ the following space-varying matrices, that will be useful in the rest of this paper:

$$
\mathcal{M}:=-\left[\alpha_{i, j}\right]_{i, j \in \underline{n}}, \mathcal{N}:=\left[\beta_{i, j}\right]_{i \in \underline{n} ; j \in \underline{m}}, \mathcal{C}:=\left[w_{i, j}\right]_{i \in \underline{p} ; j \in \underline{n}} .
$$




\section{Dynamical PROPERTIES OF THE LineARIZED MODEL}

This section focuses on dynamical properties, viz. exponential stability, reachability and observability of the linearized model (2.5)-(2.9). The fundamental observation leading to these properties is the strong relation between the infinite-dimensional linear system $\Sigma(A, B, C)$ (see e.g. [13]) and the finite-dimensional linear spacevarying system $\Sigma(\mathcal{M}, \mathcal{N}, \mathcal{C})$ (see e.g. [10]) given by $(2.10)$. This relation is observed thanks to the fact that the solution of a related operator Lyapunov equation can be built from the solution of the corresponding matrix Lyapunov equation: see Section 3.2. First let us define the controllability matrix of a matrix pair $(\mathcal{M}, \mathcal{N})$ (see [25], p. 66):

$$
Q_{c}(z):=\left[P_{0}(z) \vdots P_{1}(z) \vdots \ldots P_{n-1}(z)\right]
$$

where

$$
P_{k+1}(z)=-\mathcal{M}(z) P_{k}(z)+\frac{\mathrm{d} P_{k}}{\mathrm{~d} z}(z), \quad P_{0}(z)=\mathcal{N}(z) .
$$

The observability matrix of a matrix pair $(\mathcal{C}, \mathcal{M})$ is defined similarly (see [25], p. 66):

$$
Q_{o}(z):=\left[S_{0}(z) \vdots S_{1}(z) \vdots \ldots \vdots S_{n-1}(z)\right]
$$

where

$$
S_{k+1}(z)=-\mathcal{M}^{T}(z) S_{k}(z)+\frac{\mathrm{d} S_{k}}{\mathrm{~d} z}(z), \quad S_{0}(z)=\mathcal{C}^{T}(z) .
$$

\subsection{Exponential stability}

The first focus concerns the exponential stability of the $C_{0}$-semigroup generated by the operator $A$. In order to prove the exponential stability of this $C_{0}$-semigroup, the complete observability (see [25]) of some related matrix pair is needed.

Lemma 3.1. The following matrix Lyapunov differential equation (MLDE)

$$
-\frac{\mathrm{d} \mathcal{P}}{\mathrm{d} z}=\mathcal{P} \mathcal{M}+\mathcal{M}^{T} \mathcal{P}+I_{n}, \quad \mathcal{P}(1)=0
$$

admits a positive definite solution, viz. the observability gramian $\mathcal{P}$, on $[0,1)$.

Proof. Observe that the corresponding observability matrix of the pair $\left(I_{n}, \mathcal{M}\right)$ (see Eqs. (3.3)-(3.4)) is full rank, whence by using [25], Theorem 5 , p. 69 , the matrix pair $\left(I_{n}, \mathcal{M}\right)$ is completely observable on the interval $[0,1)$. Consequently, the MLDE (3.5) has a positive definite solution on the interval $[0,1)$ (see e.g. [10]).

By observing that the solution of the corresponding operator Lyapunov algebraic equation (OLAE) can be built from the solution of the MLDE (3.5), Lemma 3.1 leads to the following exponential stability result.

Theorem 3.1. The operator $A$ defined by (2.6)-(2.7) is the infinitesimal generator of an exponentially stable $C_{0}$-semigroup of bounded linear operators on $H$.

Proof. In view of [13], Theorem 5.3.1, p. 217, it suffices to prove that there exists a positive definite operator $P \in$ $\mathcal{L}(H)$ satisfying the following OLAE

$$
P A+A^{*} P+I=0 \text { with } P(D(A)) \subset D\left(A^{*}\right) .
$$

In view of the structure of the operator $A$, it seems natural to look for a solution of the form $P=\left[\mathcal{P}_{i, j}(z) I\right]_{i, j \in \underline{n}}$, where $I$ is the identity operator and every $\mathcal{P}_{i, j}$ is a real valued function defined on $[0,1]$ and is the $(i, j)$-entry of a symmetric matrix function $z \in[0,1] \mapsto \mathcal{P}(z) \in \mathbb{R}^{n \times n}$. The notation $\left[\mathcal{P}_{i, j}(z) I\right]_{i, j \in \underline{n}}$ is adopted here in order 
to avoid any confusion between a matrix with scalar valued entries and an (multiplication) operator matrix. By a straightforward calculation, the following identities can be shown to hold:

$$
P A=P A_{0}+P M \text { and } A^{*} P=A_{0}^{*} P+M^{*} P
$$

where the operator $A_{0}$ is the unbounded linear diagonal operator defined on its domain

$$
D\left(A_{0}\right):=\left\{x \in H: x \text { is a.c., } \frac{\mathrm{d} x}{\mathrm{~d} z} \in H \text { and } x(0)=0\right\}
$$

by

$$
A_{0}:=\left[\begin{array}{cccc}
-\frac{\mathrm{d}}{\mathrm{d} z} & 0 & \ldots & 0 \\
0 & -\frac{\mathrm{d}}{\mathrm{d} z} & \ldots & 0 \\
\vdots & \vdots & \ddots & \vdots \\
0 & 0 & \ldots & -\frac{\mathrm{d} .}{\mathrm{d} z}
\end{array}\right]
$$

and the operator $M$ is given by $M=\left[\mathcal{M}_{i, j}(z) I\right]_{i, j \in \underline{n}}$. On the other hand, it can be shown that

$$
P A_{0}+A_{0}^{*} P=\frac{\mathrm{d} P}{\mathrm{~d} z}
$$

Therefore, if the matrix $\mathcal{P}:=\left[\mathcal{P}_{i, j}(z)\right]_{i, j \in \underline{n}}$ is the positive definite solution of the MLDE (3.5), then the operator $P:=\left[\mathcal{P}_{i, j}(z) I\right]_{i, j \in \underline{n}}$ is a solution of the OLAE (3.6), since the final condition $\mathcal{P}(1)=0$ implies that $P(D(A)) \subset$ $D\left(A^{*}\right)$. Moreover this solution is positive definite. Indeed, for any $x \in H$, one has

$$
\langle P x, x\rangle_{H}=\int_{0}^{1}\left(x^{T}(z) \mathcal{P}(z) x(z)\right) \mathrm{d} z
$$

Hence one can conclude that $P$ is also positive definite since the matrix function $\mathcal{P}$ is positive definite on $[0,1]$ by Lemma 3.1 .

The following result is an obvious consequence of Theorem 3.1 (see e.g. [13]).

Corollary 3.1. The operator pair $(A, B)$ is exponentially stabilizable and the operator pair $(C, A)$ is exponentially detectable.

\subsection{Reachability and observability}

The second question concerns the reachability of $(A, B)$, where $A$ is given by (2.6)-(2.7) and $B$ is given by (2.8). Noting that the fact that the reachability operator Lyapunov algebraic equation can be related to some matrix Lyapunov differential equation leads to the following result:

Theorem 3.2. Let $A$ and $B$ be the operators defined by (2.6)-(2.7) and (2.8), respectively. Let $\mathcal{M}$ and $\mathcal{N}$ be the matrices defined by $(2.10)$. If the matrix pair $(\mathcal{M}, \mathcal{N})$ is totally controllable on $[0,1]$ then the operator pair $(A, B)$ is reachable.

Proof. First recall that the operator $A$ generates an exponentially stable $C_{0}$-semigroup. Then by [13], Theorem 4.1.23, p. 160, the extended reachability gramian, denoted by $L_{B}$, is the unique self-adjoint solution of the following OLAE:

$$
L_{B}\left(D\left(A^{*}\right)\right) \subset D(A), \quad \text { and } \quad\left[A L_{B}+L_{B} A^{*}+B B^{*}\right] x=0, x \in D\left(A^{*}\right) .
$$


In order to prove the reachability of $(A, B)$ and in view of [13], Theorem 4.1.22, p. 160, it is enough to show that the solution $L_{B}$ is positive definite. Note that by a straightforward calculation it can be shown that if the matrix $\mathcal{L}_{B}:=\left(l_{i, j}(z)\right)_{1 \leq i, j \leq n}$ is the positive definite solution of the MLDE

$$
\frac{\mathrm{d} \mathcal{L}_{B}}{\mathrm{~d} z}=\mathcal{M} \mathcal{L}_{B}+\mathcal{L}_{B} \mathcal{M}^{T}+\mathcal{N} \mathcal{N}^{T}, \mathcal{L}_{B}(0)=0,
$$

whose existence is guaranteed by the total controllability of the matrix pair $(\mathcal{M}, \mathcal{N})$, then the operator $\mathrm{七}_{B}:=$ $\left(l_{i, j}(z) I\right)_{1 \leq i, j \leq n}$ is the solution of the OLAE (3.10). Moreover $L_{B}$ is positive definite since the matrix function $\mathcal{L}_{B}$ is positive definite on $[0,1]$.

In view of [25], Theorem 3, p. 69, the following corollary is a straightforward consequence of Theorem 3.2:

Corollary 3.2. If the controllability matrix $Q_{c}(z)$, defined by equations (3.1)-(3.2), is full rank, i.e. $\operatorname{rank}\left(Q_{c}(z)\right)=n$ for all $z \in[0,1]$, then the operator pair $(A, B)$ is reachable.

By using duality and arguments similar to those used for the reachability analysis, it can be shown that the observability follows from the total observability of some related space-varying system. This is summarized in the following theorem and corollary:

Theorem 3.3. Let $A$ and $C$ be the operators defined by (2.6)-(2.7) and (2.9), respectively. Let $\mathcal{M}$ and $\mathcal{C}$ be the matrices defined by $(2.10)$. If the matrix pair $(\mathcal{C}, \mathcal{M})$ is completely observable on $[0,1]$ then the operator pair $(C, A)$ is observable.

Corollary 3.3. If the observability matrix $Q_{o}(z)$, defined by equation (3.3)-(3.4) is full rank, i.e. $\operatorname{rank}\left(Q_{o}(z)\right)=n$ for some $z \in[0,1]$, then the operator pair $(C, A)$ is observable.

\section{LQ-Optimal CONTROL DESIGN}

This section is devoted to the design of an optimal LQ-feedback (see e.g. [11,13]) for the linearized model given by (2.5)-(2.9). More precisely the aim is to find a control law which minimizes the following cost criterion:

$$
\Lambda\left(x_{0} ; u\right)=\int_{0}^{\infty}(\langle y(s), y(s)\rangle+\langle u(s), u(s)\rangle) \mathrm{d} s .
$$

First note that the fact that $A$ generates an exponentially stable $C_{0}$-semigroup (see Th. 3.1) implies that $(A, B)$ is exponentially stabilizable and $(C, A)$ is exponentially detectable.

It is well known (see e.g. [13]) that, under those conditions, the solution of this problem can be obtained by finding the nonnegative self-adjoint operator $Q_{o} \in \mathcal{L}(H)$ that solves the operator Riccati algebraic equation (ORAE), viz.

$$
\left[A^{*} Q_{o}+Q_{o} A+C^{*} C-Q_{o} B B^{*} Q_{o}\right] x=0
$$

for all $x \in D(A)$, where $Q_{o}(D(A)) \subset D\left(A^{*}\right)$.

In order to solve this equation, the following lemma, which can be easily proven by noting that the matrices $\mathcal{C}^{T} \mathcal{C}$ and $\mathcal{N N}^{T}$ are positive semi-definite (see [1], Cor. 6.7.36), is useful.

Lemma 4.1. Let us consider the following matrix Riccati differential equation (MRDE):

$$
-\frac{\mathrm{d} \Phi}{\mathrm{d} z}=\mathcal{M}^{T} \Phi+\Phi \mathcal{M}+\mathcal{C}^{T} \mathcal{C}-\Phi \mathcal{N}^{T} \Phi, \quad \Phi(1)=0 .
$$

Then the latter has a unique positive semi-definite solution on $[0,1]$. 
Now we are in a position to state the following result:

Theorem 4.1. Let us consider the linearized model (2.5)-(2.9). Let $\mathcal{N} \in \mathbb{R}^{n \times m}$ be given by (2.10) and $\Phi \in \mathbb{R}^{n \times n}$ be the solution of the MRDE (4.3). Consider

$$
\Psi(z)=:-\mathcal{N}^{T}(z) \Phi(z) \in \mathbb{R}^{m \times n} .
$$

Then $Q_{0}:=\left[\Phi_{i, j} I\right]_{i, j \in \underline{n}}$ is the unique self-adjoint positive semi-definite solution of the operator Riccati differential equation (4.2). Hence the operator

$$
K_{0}:=\left[\Psi_{i, j} I\right]_{i \in \underline{m}}, j \in \underline{n}
$$

is the optimal $L Q$-feedback and the LQ-optimal control law is given by

$$
u_{\text {opt }}(z, t)=\Psi(z) x(z, t) .
$$

Proof. Let us prove that $Q_{o}$ is the unique self-adjoint positive semi-definite solution of the ORAE (4.2). First observe that

$$
A^{*} Q_{0}=A_{0}^{*} \Phi+M^{*} \Phi \quad \text { and } \quad Q_{0} A=\Phi A_{0}+\Phi M,
$$

where $A_{0}$ is given by (3.8). By direct calculation, it can be easily shown that

$$
A_{0}^{*} \Phi+\Phi A_{0}=\left[\frac{\mathrm{d} \Phi_{i, j}}{\mathrm{~d} z} I\right]_{i, j \in \underline{n}} .
$$

On the other hand $C^{*} C=\left[\left(\mathcal{C}^{T} \mathcal{C}\right)_{i, j} I\right]_{i, j \in \underline{n}}$ and $Q_{0} B B^{*} Q_{0}=\left[\left(\Phi \mathcal{N N}^{T} \Phi\right)_{i, j} I\right]_{i, j \in \underline{n}}$. Therefore if the matrix $\Phi$ is a solution of the MRDE (4.3), then $Q_{o}=\bar{\Phi} I$ is a solution of the ORAE (4.2) since the initial condition $\Phi(1)=0$ implies that $Q_{o}(D(A)) \subset D\left(A^{*}\right)$. Moreover the fact that the MRDE (4.3) has a unique positive semi-definite solution $\Phi$ on $[0,1]$ implies that the operator $Q_{o}=\Phi I$ is the unique self-adjoint positive semi-definite solution of the ORAE (4.2).

Remark 4.1. In view of Theorem 3.2 and Corollary 3.2, if the matrix pair $(\mathcal{M}, \mathcal{N})$ is totally controllable, or equivalently the controllability matrix $Q_{c}$ is full rank on the interval $[0,1]$, then the LQ-optimal feedback $K_{0}$ can also be uniquely determined by spectral factorization (see e.g. [9]). This alternative method was successfully applied to a nonisothermal plug flow reactor in $[4,5]$.

\section{Nonlinear CLOSED-LOOP SYSTEM PERFORMANCES ANALYSIS}

This section focuses on the performances of the resulting closed-loop nonlinear system based on the implementation of the optimal LQ-feedback designed in the previous section. Here, we are interested in two questions. The first one is the asymptotic stability. The second one is the optimality of the designed LQ-control for the nonlinear system since its optimality is proved only on the linearized model with respect to a chosen quadratic criterion. Without loss of generality let us assume that the optimal LQ-feedback is computed for the linearized model around the zero equilibrium profile.

\subsection{Stability analysis}

In this subsection, we are interested in the asymptotic stability of the closed-loop nonlinear model. The latter can be described by applying the LQ-optimal state feedback $K_{0}$, given by (4.5), to the nonlinear model (2.1)-(2.2):

$$
\left\{\begin{array}{l}
\dot{x}(t)=A_{0} x(t)+N_{0}(x(t)) \\
x(0)=x_{0} \in D\left(A_{0}\right)
\end{array}\right.
$$


where the operator $A_{0}$ is the linear (unbounded) diagonal operator given by equation (3.8) and the nonlinear operator $N_{0}$ is defined on $H$ by

$$
N_{0}(x):=f\left(x, K_{0} x\right) .
$$

First we assume that the operator $\mathcal{N}_{0}$ is defined everywhere. The analysis of the following results is based on concepts and results developed e.g. in $[9,14,19,21]$.

Definition 5.1. Let $\mathcal{A}$ be an operator on a Banach space $X$ with domain $D(\mathcal{A})$.

(i) $\mathcal{A}$ is said to be dissipative if, for all $x, x^{\prime} \in D(\mathcal{A})$ and for all $\lambda>0$,

$$
\left\|x-x^{\prime}\right\| \leq\left\|\left(x-x^{\prime}\right)-\lambda\left(\mathcal{A} x-\mathcal{A} x^{\prime}\right)\right\|,
$$

or equivalently, for all $x, x^{\prime} \in D(\mathcal{A})$, there exists a bounded linear functional $f$ on $X$ such that $f\left(x-x^{\prime}\right)=\left\|x-x^{\prime}\right\|^{2}=\|f\|^{2}$ and $f\left(\mathcal{A} x-\mathcal{A} x^{\prime}\right) \leq 0$.

In addition $\mathcal{A}$ is said to be strictly dissipative if the conditions above hold with strict inequalities, for all $x, x^{\prime} \in D(\mathcal{A})$ such that $x \neq x^{\prime}$.

(ii) $\mathcal{A}$ is said to be (strictly) m-dissipative if it is (strictly) dissipative and $\mathcal{R}(I-\mathcal{A})=X$, where $\mathcal{R}(T)$ denotes the range of an operator $T$.

Theorem 5.1. Consider the closed-loop nonlinear system (5.1)-(5.2). If the operator $N_{0}$ is strictly dissipative on the Hilbert space $H$, then the operator $A_{0}+N_{0}$ is the generator of a unique nonlinear contraction semigroup $\Gamma(t)$ on $H$. Moreover, for any $x_{0} \in H$,

$$
x\left(t, x_{0}\right):=\Gamma(t) x_{0} \rightarrow 0 \text { as } t \rightarrow \infty
$$

i.e. the zero state is an asymptotically stable equilibrium state.

Proof. Observe that each diagonal entry of the operator $A_{0}$ generates an exponentially stable $C_{0}$-semigroup of contraction (see [13], Ex. 2.2.4, p. 34), whence it is m-dissipative. It follows that the operator $A_{0}$ is m-dissipative.

On the other hand $\left(I-\lambda A_{0}\right)^{-1}$ is compact. Indeed, consider the operator

$$
\mathcal{A}_{d}:=-\frac{\mathrm{d}}{\mathrm{d} z}
$$

defined on the domain

$$
D\left(\mathcal{A}_{d}\right)=\left\{x \in L^{2}(0,1): x \text { is absolutely continuous, } \frac{\mathrm{d} x}{\mathrm{~d} z} \in L^{2}(0,1) \text { and } x(0)=0\right\} .
$$

A straightforward computation shows that, for any $\lambda>0$ and for any $g \in L^{2}(0,1)$,

$$
\left(\left(I-\lambda \mathcal{A}_{d}\right)^{-1} g\right)(z)=\frac{1}{\lambda} \exp \left(-\frac{z}{\lambda}\right) \int_{0}^{z} \exp \left(\frac{\eta}{\lambda}\right) g(\eta) \mathrm{d} \eta .
$$

Using this identity, it can be shown that $\left(I-\lambda \mathcal{A}_{d}\right)^{-1}$ is a Hilbert-Schmidt operator, whence it is compact. It follows that the operator $\left(I-\lambda A_{0}\right)^{-1}$ is also compact, as a diagonal matrix of compact operators. Then, by using the strict dissipativity of $N_{0}$ the conclusion follows directly from [2], Theorem 4.3.1, pp. 59-60.

Theorem 5.2. Consider the closed-loop nonlinear system (5.1)-(5.2). Assume that the operator $N_{0}$ is Lipschitz continuous with Lipschitz constant $l_{0}$. Then, if $l_{0}<\mathrm{e}^{-1}$, the operator $A_{0}+N_{0}$ is the generator of a unique nonlinear contraction semigroup $\Gamma(t)$ on $H$. Moreover, for any $x_{0} \in H$,

$$
x\left(t, x_{0}\right):=\Gamma(t) x_{0} \rightarrow 0 \text { as } t \rightarrow \infty
$$

i.e. the zero state is an asymptotically stable equilibrium state. 
Proof. Let us denote by $\omega_{0}$ the growth bound of the $C_{0}$-semigroup $\left(\mathrm{e}^{A_{0} t}\right)_{t \geq 0}$. By using [13], Theorem 2.1.6(e), p. 18, and its proof, for all $\omega>\omega_{0}$, there exists a $t_{\omega}$ such that

$$
\frac{\log \left\|\mathrm{e}^{A_{0} t}\right\|}{t}<\omega, \quad \text { for } t \geq t_{\omega}, \text { or equivalently }\left\|\mathrm{e}^{A_{0} t}\right\| \leq \mathrm{e}^{\omega}, \text { for } t \geq t_{\omega}
$$

Note also that $\left\|\mathrm{e}^{A_{0} t}\right\|$ is bounded on every finite subinterval of $[0, \infty)$, in particular in the interval $\left[0, t_{\omega}\right]$, i.e. there exists $M_{0, \omega}>0$ such that

$$
\left\|\mathrm{e}^{A_{0} t}\right\| \leq M_{0, \omega}, \quad \forall 0 \leq t \leq t_{\omega}
$$

Then $\forall \omega \geq \omega_{0}$, there exists a constant $M_{\omega}=\left\{\begin{array}{ll}M_{0, \omega}, & \text { if } \omega>0 \\ \mathrm{e}^{-\omega t_{\omega}} M_{0, \omega} & \text { if } \omega<0\end{array}\right.$, such that

$$
\left\|\mathrm{e}^{A_{0} t}\right\| \leq M_{\omega} \mathrm{e}^{\omega t}, \text { for all } t \geq 0
$$

On the other hand, it can be shown that $\left\|\mathrm{e}^{A_{0} t}\right\|=0$, for all $t \geq 1$ and $\left\|\mathrm{e}^{A_{0} t}\right\|=1$, for all $t<1$. Then (a) the growth bound of the $C_{0}$-semigroup $\left(\mathrm{e}^{A_{0} t}\right)_{t \geq 0}$ is equal to $-\infty$, (b) the constant $t_{\omega}$ defined above is equal to 1 and (c) the constant $M_{0, \omega}$ is equal to 1 . Consequently, for all $\omega<0$

$$
\left\|\mathrm{e}^{A_{0} t}\right\| \leq M_{\omega} \mathrm{e}^{\omega t}, \text { for all } t \geq 0, \quad \text { where } M_{\omega}=\mathrm{e}^{-\omega}
$$

By using [6], Corollary 13, p. 126, the asymptotic stability follows from [6], Comment 14, p. 126, since $\sup _{\omega<0}\left\{-\omega \mathrm{e}^{\omega}\right\}=\mathrm{e}^{-1}$.

In the previous results, we have assumed that the nonlinear operator $N_{0}$ is defined everywhere. Now we are interested in the asymptotic stability of the system (5.1) when the operator $N_{0}$ is defined on a convex closed subset $F \subset H$. First the following technical concept is recalled:

Definition 5.2. Let $\mathcal{A}$ be a dissipative operator. Let $X_{0}$ be a subset of $X$. $\mathcal{A}$ is said to be in $Q\left(X_{0}\right)$ if $\overline{D(\mathcal{A})} \subset X_{0}$ and $X_{0} \subset \mathcal{R}(I-\lambda \mathcal{A})$ for all $\lambda>0$.

On the other hand, the following result, whose proof can be found in [2,6], is needed.

Lemma 5.1 ([6], Th. 16). Let $F$ be a closed convex subset of $X$. Consider a linear closed dissipative operator $A_{0}$ such that $\left(I-\lambda A_{0}\right)^{-1}$ is compact for some $\lambda>0$. Consider a Lipschitz continuous nonlinear operator $N_{0}$ on $F$. Assume that $\mathcal{A}=A_{0}+N_{0}$ is strictly dissipative and the restriction of $A_{0}$ to $D\left(A_{0}\right) \cap F$ is in $Q(F)$ and that the condition

$$
\liminf _{\lambda \rightarrow 0^{+}} \lambda^{-1} d\left(F, x+\lambda N_{0}(x)\right)=0, \text { for } x \in \overline{D(\mathcal{A})}
$$

holds, where $d(F, p)$ denotes the distance from $p \in X$ to $F$. Let $\Gamma(t)$ be the contraction semigroup generated by $\mathcal{A}$. Assume that $\bar{x}$ is an equilibrium point of $\mathcal{A}$. Then for any $x_{0} \in \overline{D(\mathcal{A})}$,

$$
x\left(t, x_{0}\right):=\Gamma(t) x_{0} \rightarrow \bar{x} \text { as } t \rightarrow \infty
$$

Now we are in a position to state the following result:

Theorem 5.3. Consider the closed-loop nonlinear model (5.1)-(5.2). Assume that the operator $N_{0}$ is Lipschitz continuous on $F$ and the operator $\mathcal{A}:=A_{0}+N_{0}$ is strictly dissipative. If $F \subset \mathcal{R}\left(I-\lambda A_{0}\right)$ for all $\lambda>0$ and if the condition

$$
\liminf _{\lambda \rightarrow 0^{+}} \lambda^{-1} d\left(F, x+\lambda N_{0}(x)\right)=0, \text { for } x \in \overline{D(\mathcal{A})}
$$

holds, then, for any $x_{0} \in \overline{D(\mathcal{A})}$,

$$
x\left(t, x_{0}\right):=\Gamma(t) x_{0} \rightarrow 0 \text { as } t \rightarrow \infty
$$

i.e. the zero state is an asymptotically stable equilibrium state. 
Proof. This result is a consequence of Lemma 5.1. Indeed, the compactness and the m-dissipativity of $A_{0}$ are shown in the proof of Theorem 5.1. On the other hand, the fact that $F \subset \mathcal{R}(I-\lambda \mathcal{A})$ for all $\lambda>0$ implies that the restriction of $A_{0}$ on $D\left(A_{0}\right) \cap F$ is in $Q(F)$ (see Def. 5.2) since $\overline{D\left(A_{0}\right) \cap F} \subset F$.

Remark 5.1. In view of (29) and since the state feedback operator $K_{0}$ is linear, it suffices to check that the function $f$ is Lipschitz continuous, in order to prove that the operator $N_{0}$ is Lipschitz continuous too. In addition, when the Hilbert space $X \times U$ is equipped with the inner product $\langle(x, u) ;(y, v)\rangle:=\langle x, y\rangle_{H}+\langle u, v\rangle_{U}$, if $l_{f}$ is a Lipschitz constant for $f$, then a Lipschitz constant for $N_{0}$ is given by $l_{0}=l_{f} \cdot \sqrt{1+\left\|K_{0}\right\|^{2}}$.

\subsection{Optimality analysis}

Obviously, the LQ-feedback designed in the previous section is not going to be optimal for the overall nonlinear system since its computation is based on the linearized model with the objective to minimize a specific quadratic criterion. This subsection in concerned with an inverse optimal control problem. This study is inspired by [17], which deals with the inverse optimal control problem for finite-dimensional systems. The question under study in this subsection is the following: what type of modification of the cost criterion can restore optimality? In this subsection it will be assumed that the function $f$ is linear with respect to the input variable i.e.

$$
f(x):=f_{0}(x)+B u .
$$

In this case the nonlinear open-loop system can be written as follows:

$$
\left\{\begin{array}{l}
\dot{x}(t)=A_{0} x(t)+B u(t)+f_{0}(x(t)) \\
x(0)=x_{0} \in D\left(A_{0}\right)
\end{array}\right.
$$

The idea is to write the generator of the latter as the sum of the linearized generator and some nonlinear operator:

$$
\left\{\begin{array}{l}
\dot{x}(t)=A x(t)+B u(t)+\tilde{f}(x(t)) \\
x(0)=x_{0} \in D\left(A_{0}\right)
\end{array}\right.
$$

where the function $\tilde{f}(x)=f_{0}(x)-J f_{0}(0) x$, where $J f_{0}(0)$ denotes the Jacobian of the function $f_{0}$ evaluated at 0 . The LQ-control law $u_{\text {opt }}$ given by (4.6) is not optimal for the nonlinear system (5.6) (except if the function $f_{0}$ is linear), but it is optimal with respect to another type of cost criterion that includes the function $\tilde{f}$ :

$$
\Lambda_{0}\left(x_{0}, u\right)=\int_{0}^{\infty} \pi(x, u) \mathrm{d} t
$$

where the function $\pi: H \times U \rightarrow \mathbb{R}$ is defined as

$$
\pi(x, u):=\langle C x, C x\rangle-2\left\langle\tilde{f}, Q_{o} x\right\rangle+\langle u, u\rangle
$$

where $Q_{0}:=\left[\Phi_{i, j} I\right]_{i, j \in \underline{n}}$ is the solution of the operator Riccati algebraic equation (4.2) and $\Phi$ is the solution of the matrix Riccati differential equation (4.3). It turns out that the asymptotic stability of the nonlinear closed-loop system (5.6) leads to the optimality of $u_{\text {opt }}$ with respect to the cost criterion (5.7).

Theorem 5.4. If zero is an asymptotically stable state for the nonlinear closed-loop system (5.6), then the LQcontrol law $u_{\text {opt }}$ given by (4.6) is optimal for the nonlinear system (5.6) with respect to the cost criterion (5.7).

Proof. In view of (5.6) and by a straightforward calculation, one gets

$$
\begin{aligned}
\Lambda_{0}\left(x_{0}, u\right) & =\int_{0}^{\infty}\left\{\langle C x, C x\rangle-2\left\langle\tilde{f}, Q_{o} x\right\rangle+\langle u, u\rangle\right\} \mathrm{d} t \\
& =\lim _{t_{1} \rightarrow \infty} \int_{0}^{t_{1}}\left\{\langle C x, C x\rangle-2\left\langle\dot{x}-A x-B u, Q_{o} x\right\rangle+\langle u, u\rangle\right\} \mathrm{d} t .
\end{aligned}
$$


Now by using the fact that $Q_{o}$ is the unique solution of the ORAE (4.2), it follows that

$$
\begin{aligned}
\Lambda_{0}\left(x_{0}, u\right) & =\lim _{t_{1} \rightarrow \infty} \int_{0}^{t_{1}}\left\{-2\left\langle\dot{x}, Q_{o} x\right\rangle+\left\|B^{*} Q_{o} x\right\|^{2}+2\left\langle u, B^{*} Q_{o} x\right\rangle+\|u\|^{2}\right\} \mathrm{d} t \\
& =\left\langle x_{0}, Q_{o} x_{0}\right\rangle+\lim _{t_{1} \rightarrow \infty}\left[-\left\langle x\left(t_{1}\right), Q_{o} x\left(t_{1}\right)\right\rangle+\int_{0}^{t_{1}}\left\{\left\langle u-K_{o} x, u-K_{o} x\right\rangle\right\} \mathrm{d} t\right] .
\end{aligned}
$$

If we replace $u$ by $u_{o p t}$ of (4.6), we obtain

$$
\Lambda_{0}\left(x_{0}, u_{o p t}\right)=\left\langle x_{0}, Q_{o} x_{0}\right\rangle-\lim _{t_{1} \rightarrow \infty}\left\langle x\left(t_{1}\right), Q_{o} x\left(t_{1}\right)\right\rangle
$$

Then

$$
\inf \Lambda_{0}\left(x_{0}, u\right)=\left\langle x_{0}, Q_{o} x_{0}\right\rangle
$$

since $x(t) \rightarrow 0$ as $t \rightarrow \infty$.

Comment 5.1. Since the operator $Q_{o}$ is nonnegative, it follows from equation (5.9) that the lower bound of the cost functional $\Lambda_{0}$, given by (5.7)-(5.8), is nonnegative. Therefore the functional $\Lambda_{0}$ is nonnegative.

In view of Theorems 5.1 and 5.2, the following corollary is an immediate consequence of Theorem 5.4:

Corollary 5.1. If the operator $\mathcal{N}_{0}$ is defined everywhere and if one of the following conditions holds:

(i) the operator $N_{0}$ is strictly dissipative;

(ii) the operator $N_{0}$ is Lipschitz continuous with Lipschitz constant $l_{0}$ and $l_{0}<\mathrm{e}^{-1}$.

Then the LQ-control law $u_{\text {opt }}$ given by (4.6) is optimal for the nonlinear system (5.6) with respect to the cost criterion (5.7).

\section{Concluding REMARKS}

In this paper, we have solved the LQ-optimal control problem for a class of first-order hyperbolic PDE's that includes convection reaction processes arising in chemical engineering. First the dynamical properties, viz. exponential stability, reachability and observability of the linearized model have been analyzed. Each of these properties can be characterized by a specific related operator Lyapunov algebraic equation whose solution can be constructed from a matrix Lyapunov differential equation. Next, an optimal control has been designed on the basis of a linearized model by using the corresponding operator Riccati algebraic equation, that can be solved via certain matrix Riccati differential equation. The computed LQ-control was applied to the nonlinear model and the closed-loop system performances were analyzed. Some simple conditions have been shown to guarantee the asymptotic stability and the optimality of the closed-loop nonlinear system with respect to a modified cost criterion.

From a physical point of view and in view of all the numerical experiments that we have made so far, we state as an educated guess the conjecture that the asymptotic stability property of the closed-loop system still holds without the condition $l_{0}<\mathrm{e}^{-1}$. However, it is not easy to prove this result by following the approach used in this paper. We leave this interesting open question as a topic for further research.

Possible topics for further research include the extension of the approach developed here to reaction-diffusion processes described by models taking axial dispersion phenomena into account, or the design of compensators based on optimal LQ-control of the estimated state. 
Acknowledgements. This paper presents research results of the Belgian Programme on Inter-University Poles of Attraction initiated by the Belgian State, Prime Minister's office for Science, Technology and Culture. The scientific responsibility rests with its authors.

The work of the first author has been partially carried out within the framework of a collaboration agreement between CESAME (Université Catholique de Louvain, Belgium) and LINMA of the Faculty of Sciences (Université Chouaib Doukkali, Morocco), funded by the Secretary of the State for Development Cooperation and by the CIUF (Conseil Interuniversitaire de la Communauté Française, Belgium).

\section{REFERENCES}

[1] H. Abou-Kandil, G. Freiling, V. Ionescu and G. Jank, Matrix Riccati Equations in Control and Systems Theory, Series: Systems \& Control: Foundations \& Applications. Birkhauser (2003).

[2] I. Aksikas, Analysis and LQ-Optimal Control of Infinite-Dimensional Semilinear Systems: Application to a Plug Flow Reactor. Ph.D. thesis, Université Catholique de Louvain, Belgium (2005).

[3] I. Aksikas, J. Winkin and D. Dochain, Stability analysis of an infinite-dimensional linearized plug flow reactor model, in Proceedings of the 43rd IEEE Conference on Decision and Control, CDC (2004) 2417-2422.

[4] I. Aksikas, J. Winkin and D. Dochain, LQ-optimal feedback regulation of a nonisothermal plug flow reactor infinite-dimensional model. Int. J. Tomography \& Statistics 5 (2007) 73-78.

[5] I. Aksikas, J. Winkin and D. Dochain, Optimal LQ-feedback regulation of a nonisothermal plug flow reactor model by spectral factorization. IEEE Trans. Automat. Control 52 (2007) 1179-1193.

[6] I. Aksikas, J. Winkin and D. Dochain, Asymptotic stability of infinite-dimensional semilinear systems: application to a nonisothermal reactor. Systems Control Lett. 56 (2007) 122-132.

[7] V. Barbu, Analysis and Control of Nonlinear Infinite Dimensional Systems. Boston: Academic Press (1993).

[8] A. Bressan, Hyperbolic Systems of Conservation Laws: The One-Dimensional Cauchy Problem. Oxford University Press (2000).

[9] H. Brezis, Opéateurs Maximaux Monotones et Semi-groupes de Contractions dans les Espaces de Hilbert, Mathematics Studies. North-Holland (1973).

[10] F.M. Callier and C.A. Desoer, Linear System Theory. Springer-Verlag, New York (1991).

[11] F.M. Callier and J. Winkin, LQ-optimal control of infinite-dimensional systems by spectral factorization. Automatica 28 (1992) $757-770$.

[12] P.D. Christofides, Nonlinear and Robust Control of Partial Differential Equation Systems: Methods and Application to Transport-Reaction Processes. Birkhauser, Boston (2001).

[13] R.F. Curtain and H.J. Zwart, An Introduction to Infinite-Dimensional Linear Systems Theory. Springer-Verlag, New York (1995).

[14] C.M. Dafermos and M. Slemrod, Asymptotic behavior of nonlinear contraction semigroups. J. Funct. Anal. 13 (1973) 97-106.

[15] D. Dochain, Contribution to the Analysis and Control of Distributed Parameter Systems with Application to (Bio)chemical Processes and Robotics. Thèse d'Agrégation de l'Enseignement Supérieur, Université Catholique de Louvain, Louvain-la-Neuve, Belgium (1994).

[16] G.F. Froment and K.B. Bischoff, Chemical Reactor Analysis and Design. 2nd edition, John Wiley, New York (1990).

[17] M. Ikeda and D.D. Siljak, Optimality and robustness of linear quadratic control for nonlinear systems. Automatica 26 (1990) 499-511.

[18] M. Laabissi, M.E. Achhab, J. Winkin and D. Dochain, Trajectory analysis of nonisothermal tubular reactor nonlinear models. Systems Control Lett. 42 (2001) 169-184.

[19] V. Lakshmikantham and S. Leela, Nonlinear Differential Equations in Abstract Spaces. Pergamon, Oxford (1981).

[20] I. Lasiecka and R. Triggiani, Control Theory for Partial Differential Equations: Continuous and Approximation Theories, Volume II: Abstract Hyperbolic-like Systems over a Finite Time Horizon. Cambridge University Press (2000).

[21] Z. Luo, B. Guo and O. Morgül, Stability and Stabilization of Infinite Dimensional Systems with Applications. Springer-Verlag, London (1999).

[22] R.H. Martin, Nonlinear Operators and Differential Equations in Banach spaces. John Wiley \& Sons, New York (1976).

[23] A. Pazy, Semigroups of Linear Operators and Application to Partial Differential Equations, Appl. Math. Sci. 44. SpringerVerlag, New York (1983).

[24] W.H. Ray, Advanced Process Control, Series in Chemical Engineering. Butterworth, Boston (1981).

[25] L.M. Silverman and H.E. Meadows, Controllability and observability in time-variable linear systems. J. SIAM Control 5 (1967) $64-73$. 